\title{
Erratum to: Objection to postoperative radiation therapy in breast cancer with one to three lymph nodes involvements
}

\author{
Takashi Ishikawa $^{1} \cdot$ Hiroshi Kaise ${ }^{1} \cdot K$ imito $\operatorname{Yamada}^{1} \cdot \operatorname{Mari} \operatorname{Hosonaga}^{1} \cdot$ \\ Takashi Chishima ${ }^{2} \cdot$ Kazutaka Narui $^{3}$ - Akimitsu Yamada ${ }^{3}$. Sadatoshi Sugae ${ }^{4}$. \\ Yasushi Ichikawa $^{4} \cdot$ Mitsuyoshi Ota $^{5} \cdot$ Miwako Nozaki $^{6} \cdot$ Ryuji Mikami $^{7}$ • \\ Koichi Tokuuye ${ }^{7}$
}

Published online: 7 July 2017

(C) The Japanese Breast Cancer Society 2017

\section{Erratum to: Breast Cancer (2017) 24:496-501 \\ DOI 10.1007/s12282-016-0749-5}

The correct name of the 11th author should be "Miwako Nozaki", and not "Miyako Nozaki" as given in the original publication of the article.

The online version of the original article can be found under doi:10.1007/s12282-016-0749-5.

Takashi Ishikawa

tishik55@gmail.com

1 Department of Breast Oncology and Surgery, Tokyo Medical University, 6-7-1 Nishishinjuku, Shinjuku, Tokyo 160-0023, Japan

2 Department of Breast Surgery, Yokohama Rosai Hospital, 3211, Kozukuecho, Kohokuku, Yokohama 222-0036, Japan

3 Department of Breast and Thyroid Surgery, Yokohama City University Medical Center, Yokohama 232-0024, Japan

4 Department of Clinical Oncology and Gastrointestinal Surgery, School of Medicine, Yokohama City University, Yokohama 232-0024, Japan

5 Department of Gastroenterology, Yokohama City University Medical Center, Yokohama 232-0024, Japan

6 Department of Radiology, Dokkyo Medical University Koshigaya Hospital, 2-1-50, Minamikoshigaya, Koshigaya, Saitama 343-8555, Japan

7 Department of Radiology, Tokyo Medical University, 6-7-1 Nishishinjuku, Shinjuku, Tokyo 160-0023, Japan 